\title{
Principal Component Noise Filtering for NAST-I Radiometric Calibration
}

\author{
Jialin Tian ${ }^{\mathrm{a}}$ and William L. Smith, Sr. ${ }^{\mathrm{b}}$ \\ aNASA Langley Research Center, MS 472, Hampton, VA, USA 23681-2199; \\ ${ }^{\mathrm{b}}$ Hampton University/University of Wisconsin-Madison, Hampton, VA USA 23668
}

\begin{abstract}
The National Polar-orbiting Operational Environmental Satellite System (NPOESS) Airborne Sounder TestbedInterferometer (NAST-I) instrument is a high-resolution scanning interferometer that measures emitted thermal radiation between 3.3 and 18 microns. The NAST-I radiometric calibration is achieved using internal blackbody calibration references at ambient and hot temperatures. In this paper, we introduce a refined calibration technique that utilizes a principal component (PC) noise filter to compensate for instrument distortions and artifacts, therefore, further improve the absolute radiometric calibration accuracy. To test the procedure and estimate the PC filter noise performance, we form dependent and independent test samples using odd and even sets of blackbody spectra. To determine the optimal number of eigenvectors, the PC filter algorithm is applied to both dependent and independent blackbody spectra with a varying number of eigenvectors. The optimal number of PCs is selected so that the total root-mean-square (RMS) error is minimized. To estimate the filter noise performance, we examine four different scenarios: apply PC filtering to both dependent and independent datasets, apply PC filtering to dependent calibration data only, apply PC filtering to independent data only, and no PC filters. The independent blackbody radiances are predicted for each case and comparisons are made. The results show significant reduction in noise in the final calibrated radiances with the implementation of the PC filtering algorithm.
\end{abstract}

Keywords: NAST-I, radiometric calibration, principal component (PC) noise filtering, Fourier transform spectrometer (FTS), remote sensing

\section{INTRODUCTION}

The National Polar-orbiting Operational Environmental Satellite System (NPOESS) Airborne Sounder TestbedInterferometer (NAST-I) instrument is a high-resolution scanning interferometer that measures emitted thermal radiation between 3.3 and 18 microns. The raw interferogram measurements are radiometrically and spectrally calibrated to produce radiance spectra, which are further processed to obtain temperature and water vapor profiles of the Earth's atmosphere via retrieval algorithms. NAST-I produces sounding data with 2 kilometer resolution (at nadir) across a 40 kilometer ground swath from a nominal altitude of 20 kilometers onboard a NASA ER-2 aircraft or similar coverage from the PROTEUS aircraft.

The NAST-I radiometric calibration is achieved using internal blackbody calibration references at ambient and hot temperatures. In this paper, we introduce a refined calibration technique that utilizes a principal component (PC) noise filter to compensate for instrument distortions and artifacts, therefore, further improve the absolute radiometric calibration accuracy. ${ }^{1,2}$ The calibration procedure is summarized in the following steps: the raw interferograms are partitioned into 30-minute segments for calibration purposes; a PC noise filtering algorithm is applied to hot blackbody (HBB) and ambient blackbody (ABB) calibration reference spectra; using the filtered HBB and ABB data, the responsivity and offset coefficients are computed and applied to PC-filtered Earth scene spectra, which results in calibrated scene radiances.

To test the procedure and estimate the PC filter noise performance, we form dependent and independent test samples by using odd and even sets of blackbody spectra. To determine the optimal number of eigenvectors, the PC filter algorithm is applied to both dependent and independent blackbody spectra with a varying number of eigenvectors. The responsivity and offset coefficients are computed from filtered dependent spectra, and then applied to the filtered independent spectra, from which, we can predict the independent blackbody radiances. The total root-mean-square (RMS) error as a function of eigenvectors is evaluated by comparing the predicted blackbody radiances with ideal Planck radiances. The optimal number of PCs is selected so that the total RMS value is minimized. To see the filtering effect on the calibration noise performance, we examine four different scenarios: apply PC filtering to both dependent

*Jialin.Tian-1@nasa.gov; phone (757)864-3698 
and independent datasets, apply PC filtering to dependent calibration data only, apply PC filtering to independent data only, and no PC filters. The independent scene radiances are predicted for each case and comparisons are made. The results show significant reduction in noise in the final calibrated radiances with the implementation of the PC filtering algorithm.

\section{PRINCIPAL COMPONENT NOISE FILTERING}

In this section, we describe the PC filtering algorithm that will be employed to reduce noise for any given group of spectra. First we define NAST-I data in matrix forms, and then introduce the fundamental filtering technique.

\subsection{Data Definition}

Each NAST-I data measurement set contains thirteen atmospheric scene measurements, one ABB calibration reference, and one HBB calibration reference. Given

$$
N_{i, j, m}^{C_{f}}(k) \text { and } N_{i, j, m}^{C_{r}}(k)
$$

as the atmospheric scene spectra for forward $\left(C_{f}\right)$ and reverse scans $\left(C_{r}\right)$, respectively, where $k=1, \ldots, K$ denotes the spectral channel indices, $i=1, \ldots, I$ represents the set number, $j=1, \ldots, J$ denotes the scene index, and $m=1, \ldots, M$ denotes the measurement scan number. Similarly, we define the ABB forward scans $\left(A_{f}\right)$, ABB reverse scans $\left(A_{r}\right)$, HBB forward scans $\left(H_{f}\right)$, and HBB reverse scans $\left(H_{r}\right)$ as

$$
N_{i, m}^{A_{f}}(k), N_{i, m}^{A_{r}}(k), N_{i, m}^{H_{f}}(k), \text { and } N_{i, m}^{H_{r}}(k),
$$

respectively.

To apply the PC noise filter, we combine 120 sets (a 30-minute segment) of spectra to form supersets. Define $\alpha=1,2, \ldots,\left\lfloor\frac{I}{120}\right\rfloor$ as the superset index and $s=1, \ldots, S$ as the new measurement scan number. The notation for the new scene spectra can be written as

$$
N_{\alpha, j, s}^{C_{f}}(k) \text { and } N_{\alpha, j, s}^{C_{r}}(k)
$$

similarly, we define the new ABB forward scans, ABB reverse scans, HBB forward scans, and HBB reverse scans as

$$
N_{\alpha, s}^{A_{f}}(k), N_{\alpha, s}^{A_{r}}(k), N_{\alpha, s}^{H_{f}}(k), \text { and } N_{\alpha, s}^{H_{r}}(k),
$$

respectively.

\subsection{PC Noise Filter Algorithm}

Once we have established the NAST-I data representation, the PC filtering method can be outlined as the following. Suppose $N_{\beta, \sigma, \lambda}(k)$ represents the spectra for any given superset $\beta$ for a particular scene $\sigma$ with $\lambda=\Lambda$ scans, its equivalent matrix form can be written as

The sample mean of $\mathbf{N}$ is

$$
N_{\beta, \sigma, \lambda}(k) \equiv \underset{\Lambda \times K}{\mathbf{N}}=\left[\begin{array}{cccc}
N_{1,1} & N_{1,2} & \ldots & N_{1, K} \\
N_{2,1} & N_{2,2} & \ldots & N_{2, K} \\
\vdots & & \ddots & \\
N_{\Lambda, 1} & N_{\Lambda, 2} & \ldots & N_{\Lambda, K}
\end{array}\right] \text {. }
$$

$$
n_{\beta, \sigma}(k)=\frac{1}{\Lambda} \sum_{\lambda=1}^{\Lambda} N_{\beta, \sigma, \lambda}(k) \equiv \underset{1 \times K}{\mathbf{n}} .
$$


Using singular value decomposition (SVD), $\mathbf{N}$ can be factorized into

$$
\mathbf{N}=\mathbf{U D P}^{* T},
$$

where $* T$ denotes conjugate transpose; $\mathbf{D}=\operatorname{diag}\left\{d_{1}, \ldots, d_{Z}\right\}$, in which $d_{1} \geq \ldots \geq d_{Z} \geq 0$ and $Z=\min \{\Lambda, K\}$. From which, the projection of the original dataset is expressed as

The PC filtered spectra can be derived using

$$
\mathbf{S}=\mathbf{D}^{* T} \mathbf{N} .
$$

$$
\widehat{\mathbf{N}}=\mathbf{P}_{e^{*}} \mathbf{S}+\mathbf{n},
$$

where $e^{*}$ is the optimal number of eigenvectors employed by the PC filter. To simplify the notation, we can write the PC filtering operation described above as $\widehat{\mathbf{N}}_{e^{*}}=p c f_{e^{*}}\{\mathbf{N}\}$, where $p c f_{e^{*}}\{\cdot\}$ denotes the NAST-I PC-filter operator.

\section{PC FILTER NOISE PERFORMANCE ESTIMATION}

Previously we have introduced the underlining PC filtering method. In this section, we discuss the procedure for testing and estimating the filter performance, which is outlined in Figure 1. First, the dependent and independent test samples are formed using odd and even sets of blackbody spectra, then we examine four different scenarios: apply PC filtering to both dependent and independent datasets, apply PC filtering to dependent calibration data only, apply PC filtering to independent data only, and no PC filters. The independent blackbody radiances are predicted for each case and comparisons are made using the theoretical Plank radiances as references.

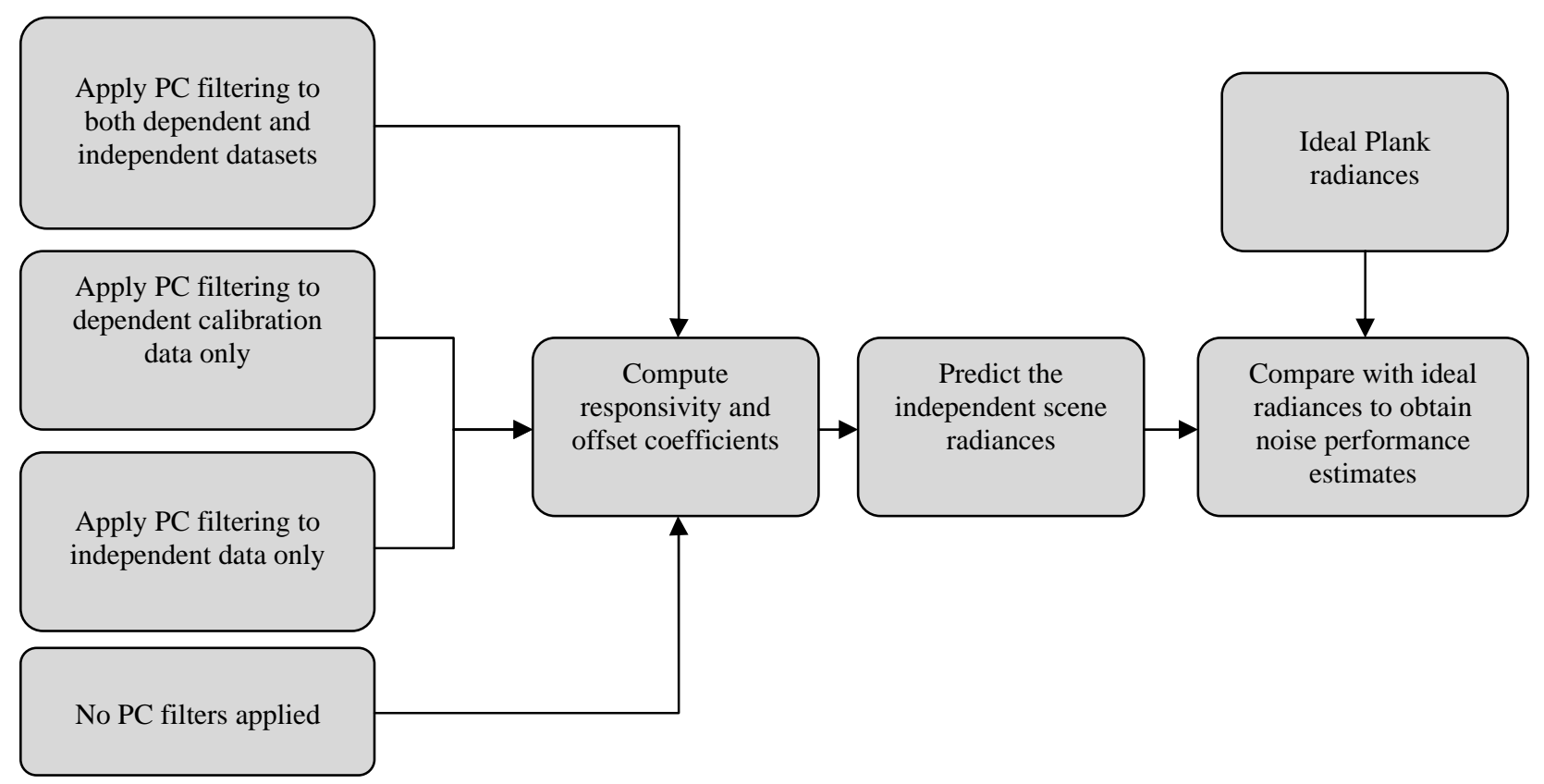

Figure 1. PC filtering noise estimation procedure.

\subsection{Dependent and Independent Test Datasets}

The dependent and independent test samples are formed using odd and even sets of blackbody spectra. We define the ABB forward dependent and independent spectral matrices within any given superset $\beta$ as

and

$$
\mathbf{N}^{A_{f_{d}}} \equiv N_{l, t}^{A_{f_{d}}}(k)=N_{\mathcal{O}\{i\}, t}^{A_{f}}(k)
$$




$$
\mathbf{N}^{A_{f_{i}}} \equiv N_{l, t}^{A_{f_{i}}}(k)=N_{\mathcal{E}\{i\}, t}^{A_{f}}(k),
$$

where the new scan index is $t=1,2, \ldots, T ; T=\frac{S}{2}$, the new set index is $l=1,2, \ldots, 60$, and the odd and even sets are written as $\mathcal{O}\{\cdot\}$ and $\mathcal{E}\{\cdot\}$. Similarly, we define the ABB reverse dependent and independent matrices as $\mathbf{N}^{A_{r_{d}}}$ and $\mathbf{N}^{A_{r_{i}}}$ respectively. By applying the same notation to HBB test matrices, we obtain $\mathbf{N}^{H_{f_{d}}}, \mathbf{N}^{H_{f_{i}}}, \mathbf{N}^{H_{r_{d}}}$, and $\mathbf{N}^{H_{r_{i}}}$. Next, we compute the 2-point moving average of the dependent sets so that they can be used as close approximations in time of the independent test sets. The moving average operation performed on ABB forward dependent dataset can be written as

$$
\overline{\mathbf{N}}^{A_{f_{d}}} \equiv \bar{N}_{l, t}^{A_{f_{d}}}(k)=\frac{1}{P} \sum_{p=0}^{P-1} N_{(l+p), t}^{A_{f_{d}}}(k) \text { for } l=1,2, \ldots, 60
$$

where $P=2$. Similarly, we obtain $\overline{\mathbf{N}}^{A_{r_{d}}}, \overline{\mathbf{N}}^{H_{f_{d}}}$, and $\overline{\mathbf{N}}^{H_{r_{d}}}$.

\subsection{Determination of the Optimal Number of Eigenvectors}

Next, we need to determine the optimal number of eigenvectors needed to filter the test datasets. Using the PC algorithm described in the previous section, we apply PC filter operation to dependent and independent blackbody spectra with a varying number of eigenvectors, $e$, for instance, $\widehat{\overline{\mathbf{N}}}_{e}^{A_{f_{d}}}=p c f_{e}\left\{\overline{\mathbf{N}}^{A_{f_{d}}}\right\}$ and $\widehat{\mathbf{N}}_{e}^{A_{f_{i}}}=p c f_{e}\left\{\mathbf{N}^{A_{f_{i}}}\right\}$. Similarly, we apply the PC algorithm to the remaining blackbody spectra matrices to obtain $\widehat{\overline{\mathbf{N}}}_{e}^{A_{r_{d}}}, \widehat{\overline{\mathbf{N}}}_{e}^{H_{f_{d}}}, \widehat{\overline{\mathbf{N}}}_{e}^{H_{r_{d}}}, \widehat{\mathbf{N}}_{e}^{A_{r_{i}}}, \widehat{\mathbf{N}}_{e}^{H_{f_{i}}}$, and $\widehat{\mathbf{N}}_{e}^{H_{r_{i}}}$. Next, we compute the forward responsivity and offset coefficients using the filtered dependent spectra

$$
\widehat{\mathbf{R}}_{e}^{f_{d}}=\frac{\widehat{\overline{\mathbf{N}}}_{e}^{H_{f_{d}}}-\widehat{\overline{\mathbf{N}}}_{e}^{A_{f_{d}}}}{\mathbf{B}^{H_{f}}-\mathbf{B}^{A_{f}}}
$$

and

$$
\widehat{\mathbf{O}}_{e}^{f_{d}}=\frac{\widehat{\overline{\mathbf{N}}}_{e}^{A_{f_{d}}} * \mathbf{B}^{H_{f}}-\widehat{\overline{\mathbf{N}}}_{e}^{H_{f_{d}}} * \mathbf{B}^{A_{f}}}{\widehat{\overline{\mathbf{N}}}_{e}^{H_{f_{d}}}-\widehat{\overline{\mathbf{N}}}_{e}^{A_{f_{d}}}}
$$

respectively, where $\mathbf{B}^{H_{f}, A_{f}}$ denotes the ideal Planck radiances at hot and ambient temperatures. We can then predict the forward independent radiances to be

$$
\widehat{\hat{\mathbf{L}}}_{e}^{A_{f}}=\Re\left\{\frac{\widehat{\mathbf{N}}_{e}^{A_{f_{i}}}}{\widehat{\mathbf{R}}_{e}^{f_{d}}}-\widehat{\mathbf{O}}_{e}^{f_{d}}\right\}
$$

and

$$
\widehat{\hat{\mathbf{L}}}_{e}^{H_{f}}=\Re\left\{\frac{\widehat{\mathbf{N}}_{e}^{H_{f_{i}}}}{\widehat{\mathbf{R}}_{e}^{f_{d}}}-\widehat{\mathbf{O}}_{e}^{f_{d}}\right\},
$$

in which $\Re\{\cdot\}$ denotes the real part of the complex radiance. Repeat the same process to obtain the reverse responsivity, offset, and predicted independent radiances as $\widehat{\mathbf{R}}_{e}^{r_{d}}, \widehat{\mathbf{O}}_{e}^{r_{d}}, \widehat{\hat{\mathbf{L}}}_{e}^{A_{r}}$, and $\widehat{\hat{\mathbf{L}}}_{e}^{H_{r}}$. To compute the total minimum mean square error, we can merge the forward and reverse radiances at ambient and hot temperatures into a single matrix, for example, $\widehat{\hat{\mathbf{L}}}_{e}=\left\{\widehat{\hat{\mathbf{L}}}_{e}^{A_{f}}, \widehat{\hat{\mathbf{L}}}_{e}^{A_{r}}, \widehat{\hat{\mathbf{L}}}_{e}^{H_{f}}, \widehat{\widehat{\mathbf{L}}}_{e}^{H_{r}}\right\}$, where $e$ is the full rank of the radiance matrix. Define the Planck matrix as $\mathbf{B}=\left\{\mathbf{B}^{A_{f}}, \mathbf{B}^{A_{r}}, \mathbf{B}^{H_{f}}, \mathbf{B}^{H_{r}}\right\}$. The difference between predicted independent radiances and ideal blackbody spectra is $\widehat{\mathbf{E}}_{e}=\widehat{\hat{\mathbf{L}}}_{e}-\mathbf{B}$. Define the RMS error spectrum as

$$
\hat{\hat{\epsilon}}_{e}(k)=\sqrt{\frac{1}{T} \sum_{t=1}^{T}\left[\widehat{\hat{E}}_{e, t}(k)-\overline{\widehat{\hat{E}}_{e}(k)}\right]^{2}},
$$

where $\overline{\widehat{E}}_{e}(k)=\frac{1}{T} \sum_{t=1}^{T} \widehat{\widehat{E}}_{e, t}(k)$ is the sample mean. The optimal number of eigenvectors, $e^{*}$, is determined so that the RMS error is minimized across the entire spectral band. For instance 


\subsection{Noise Performance Estimation}

$$
e^{*}=\min _{e}\left|\sum_{k=1}^{K} \hat{\hat{\epsilon}}_{e}(k)\right| .
$$

To see the filtering effect on the calibration noise performance, we examine four different scenarios. In the first case, the filtered dependent data are applied to filtered independent data. This is the same scenario described in the previous section where we derived the optimal number of eigenvectors $e^{*}$ using the iterative method. The predicted independent radiances can be written as $\widehat{\hat{\mathbf{L}}}_{e^{*}}$, its corresponding RMS error is expressed as $\hat{\hat{\epsilon}}_{e^{*}}(k)$.

In the second case, to see the filtering effect on the independent data, we can apply the PC filter algorithm to only the independent spectra while keeping the dependent data unfiltered. We compute the unfiltered forward responsivity and offset coefficients from

and

$$
\mathbf{R}^{f_{d}}=\frac{\overline{\mathbf{N}}^{H_{f_{d}}}-\overline{\mathbf{N}}^{A_{f_{d}}}}{\mathbf{B}^{H_{f}}-\mathbf{B}^{A_{f}}}
$$

$$
\mathbf{O}^{f_{d}}=\frac{\overline{\mathbf{N}}^{A_{f_{d}}} * \mathbf{B}^{H_{f}}-\overline{\mathbf{N}}^{H_{f_{d}}} * \mathbf{B}^{A_{f}}}{\overline{\mathbf{N}}^{H_{f_{d}}}-\overline{\mathbf{N}}^{A_{f_{d}}}} .
$$

The forward independent radiances are predicted from filtered independent spectra using unfiltered responsivity and offset. The results are written as

and

$$
\widetilde{\mathbf{L}}_{e^{*}}^{A_{f}}=\Re\left\{\frac{\widehat{\mathbf{N}}_{e^{*}}^{A_{f_{i}}}}{\mathbf{R}^{f_{d}}}-\mathbf{O}^{f_{d}}\right\}
$$

$$
\widetilde{\hat{\mathbf{L}}}_{e^{*}}^{H_{f}}=\Re\left\{\frac{\widehat{\mathbf{N}}_{e^{*}}^{H_{f_{i}}}}{\mathbf{R}^{f_{d}}}-\mathbf{O}^{f_{d}}\right\} .
$$

The combined radiance matrix is $\widetilde{\widehat{\mathbf{L}}}_{e^{*}}=\left\{\widetilde{\widehat{\mathbf{L}}}_{e^{*}}^{A_{f}}, \widetilde{\widehat{\mathbf{L}}}_{e^{*}}^{A_{r}}, \widetilde{\widehat{\mathbf{L}}}_{e^{*}}^{H_{f}}, \widetilde{\widehat{\mathbf{L}}}_{e^{*}}^{H_{r}}\right\}$ and $\widetilde{\widehat{\mathbf{E}}}_{e^{*}}=\widetilde{\widehat{\mathbf{L}}}_{e^{*}}-\mathbf{B}$. We can derive its corresponding RMS error to be $\tilde{\hat{\epsilon}}_{e^{*}}(k)$. Figure 2 illustrates the RMS noises in brightness temperature for cases 1 and 2.

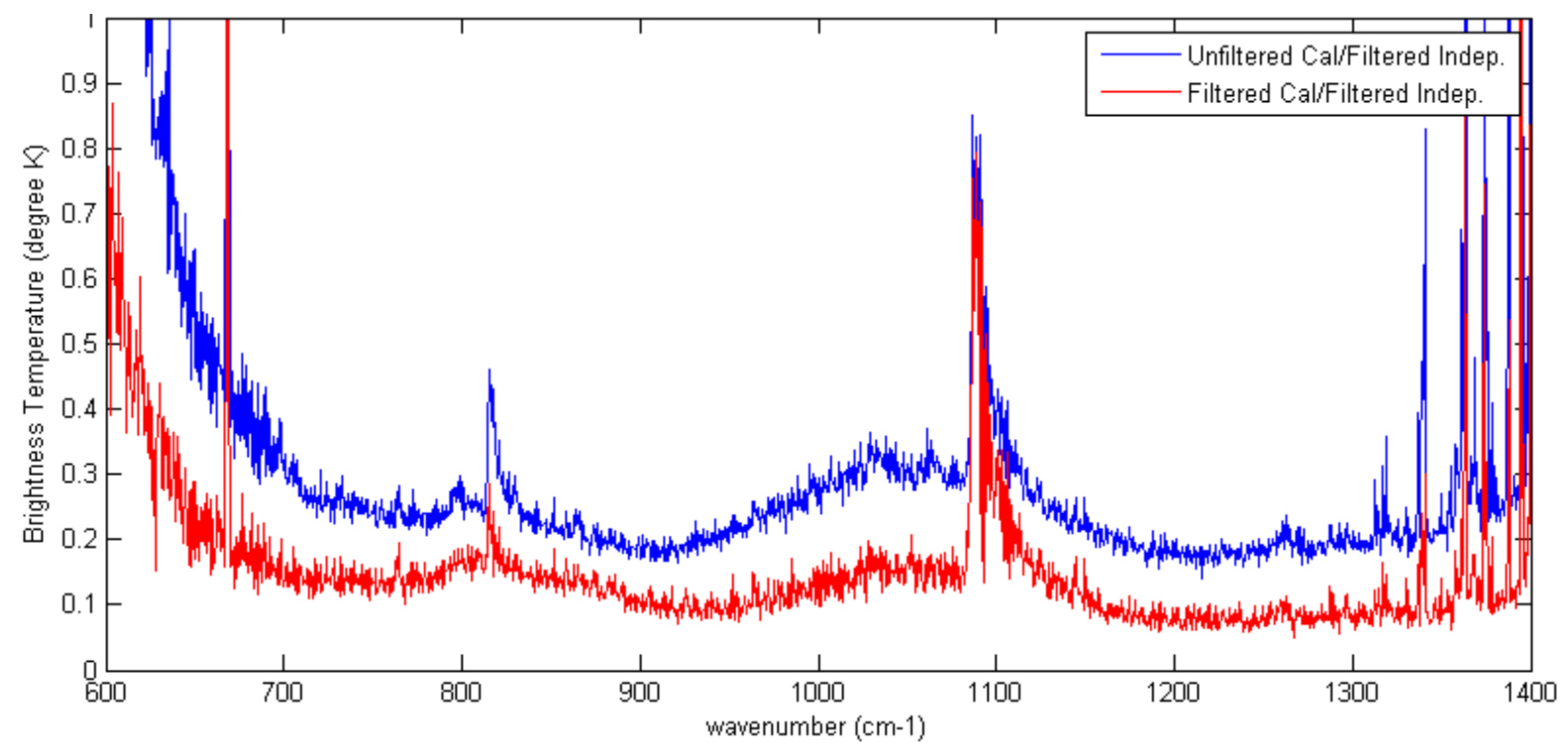

Figure 2. A comparison of RMS noises in BB brightness temperature for cases 1 \& 2 (scan average). 
In the third case, the filtered dependent data are applied to unfiltered independent data to illustrate the filtering effect on the dependent data. Similar to previous calculations, we compute the filtered forward responsivity and offset matrices from

and

$$
\widehat{\mathbf{R}}_{e^{*}}^{f_{d}}=\frac{\widehat{\mathbf{N}}_{e^{*}}^{H_{f_{d}}}-\widehat{\mathbf{N}}_{e^{*}}^{A_{f_{d}}}}{\mathbf{B}^{H_{f}}-\mathbf{B}^{A_{f}}}
$$

$$
\widehat{\mathbf{O}}_{e^{*}}^{f_{d}}=\frac{\widehat{\mathbf{N}}_{e^{*}}^{A_{f_{d}}} * \mathbf{B}^{H_{f}}-\widehat{\overline{\mathbf{N}}}_{e^{*}}^{H_{f_{d}}} * \mathbf{B}^{A_{f}}}{\widehat{\mathbf{N}}_{e^{*}}^{H_{f_{d}}}-\widehat{\overline{\mathbf{N}}}_{e^{*}}^{A_{f_{d}}}} .
$$

The forward independent radiances are predicted from unfiltered independent spectra using filtered responsivity and offset. The results are written as

and

$$
\widehat{\widetilde{\mathbf{L}}}_{e^{*}}^{A_{f}}=\Re\left\{\frac{\mathbf{N}^{A_{f_{i}}}}{\widehat{\mathbf{R}}_{e^{*}}^{f_{d}}}-\widehat{\mathbf{O}}_{e^{*}}^{f_{d}}\right\}
$$

$$
\widehat{\widehat{\mathbf{L}}}_{e^{*}}^{H_{f}}=\Re\left\{\frac{\mathbf{N}^{H_{f_{i}}}}{\widehat{\mathbf{R}}_{e^{*}}^{f_{d}}}-\widehat{\mathbf{O}}_{e^{*}}^{f_{d}}\right\} .
$$

The combined radiance matrix is $\widehat{\widetilde{\mathbf{L}}}_{e^{*}}=\left\{\widehat{\widetilde{\mathbf{L}}}_{e^{*}}^{A_{f}}, \widehat{\widetilde{\mathbf{L}}}_{e^{*}}^{A_{r}}, \widehat{\widetilde{\mathbf{L}}}_{e^{*}}^{H_{f}}, \widehat{\widetilde{\mathbf{L}}}_{e^{*}}^{H_{r}}\right\}$ and $\widehat{\widetilde{\mathbf{E}}}_{e^{*}}=\widehat{\widetilde{\mathbf{L}}}_{e^{*}}-$ B. We can derive its corresponding RMS error to be $\hat{\tilde{\epsilon}}_{e^{*}}(k)$.

In the last case, the calibration was performed without PC filters. Here we compute the unfiltered radiances to be

and

$$
\mathbf{L}^{A_{f}}=\Re\left\{\frac{\mathbf{N}^{A_{f_{i}}}}{\mathbf{R}^{f_{d}}}-\mathbf{O}^{f_{d}}\right\}
$$

$$
\mathbf{L}^{H_{f}}=\Re\left\{\frac{\mathbf{N}^{H_{f_{i}}}}{\mathbf{R}^{f_{d}}}-\mathbf{O}^{f_{d}}\right\} .
$$

The combined radiance matrix is $\mathbf{L}=\left\{\mathbf{L}^{A_{f}}, \mathbf{L}^{A_{r}}, \mathbf{L}^{H_{f}}, \mathbf{L}^{H_{r}}\right\}$ and $\mathbf{E}=\mathbf{L}-\mathbf{B}$. Its corresponding RMS error is $\epsilon(k)$. Figure 3 illustrates the RMS noises in brightness temperature for cases 3 and 4.

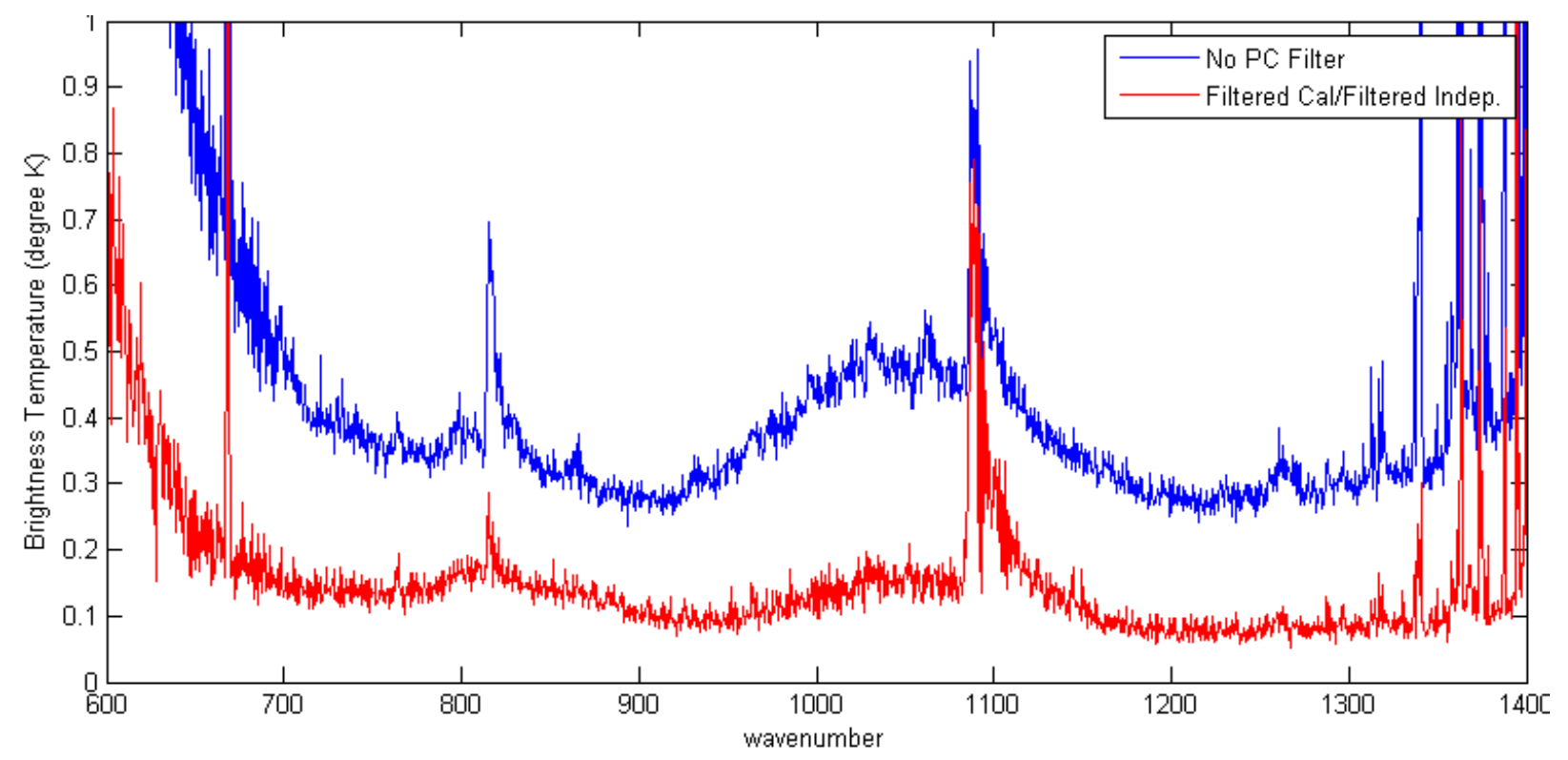

Figure 3. A comparison of RMS noises in BB brightness temperature for cases 3 \& 4 (scan average). 


\section{COMPLEX PC RADIOMETRIC CALIBRATION}

In the previous section, we have presented the procedure for testing and estimating the filter performance using blackbody spectra as dependent and independent test simples. In this section, we apply the PC filtering method to actual Earth scene spectra. Figure 4 describes the complex PC radiometric calibration process. Initially, the spectra are partitioned into 30-minute segments according to their scan directions, and then we apply the filtering algorithm to both the blackbody calibration data and Earth scene data using the optimal number of eigenvectors. The responsivity and offset coefficients are computed and the calibrated radiances are obtained. The same process is repeated for all data segments. The calibrated radiances for all segments are then regrouped into their original format.

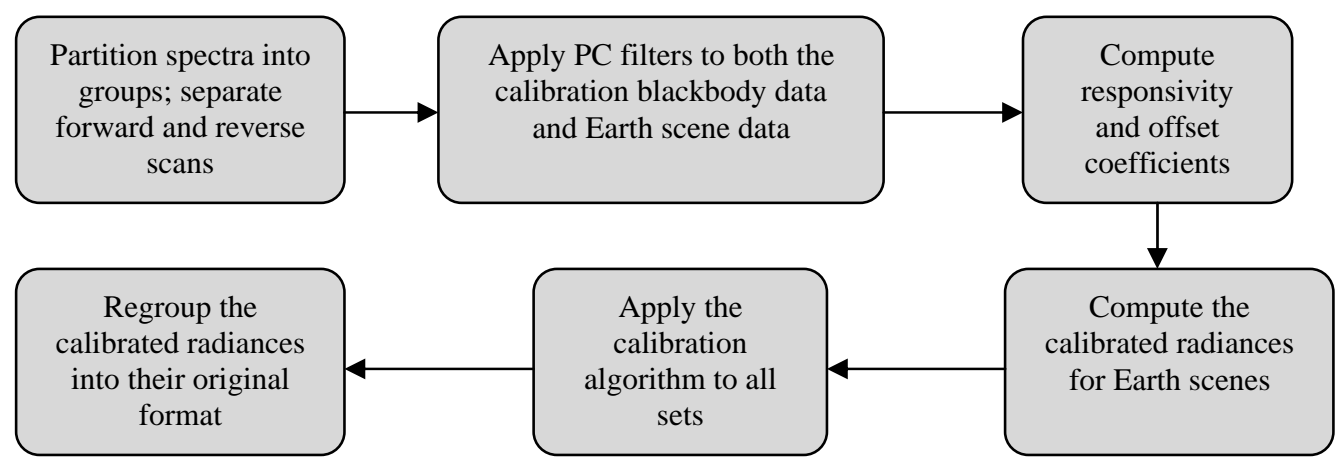

Figure 4. Complex PC radiometric calibration procedure.

\subsection{Optimal Eigenvectors for Atmospheric Scene Spectra}

Before we can implement the PC filter to the atmospheric scene spectra, the optimal number of eigenvectors must be determined. Define the ABB and HBB forward and reverse matrices as $\mathbf{N}^{A_{f}, A_{r}, H_{f}, H_{r}} \equiv N_{\alpha, s}^{A_{f}, A_{r}, H_{f}, H_{r}}(k)$, the forward and reverse scenes as $\mathbf{N}^{C_{f}, C_{r}} \equiv N_{\alpha, j, s}^{C_{f}, C_{r}}(k)$, and the PC filtered blackbody calibration matrices as $\widehat{\mathbf{N}}_{e^{*}}^{A_{f}, A_{r}, H_{f}, H_{r}}=p c f_{e^{*}}\left\{\mathbf{N}^{A_{f}, A_{r}, H_{f}, H_{r}}\right\}$. We derive the radiances for two cases. In the first case, we apply PC filtering to the calibration data but leaving the Earth scene data unfiltered. In this case, the filtered forward responsivity and offset coefficients are

and

$$
\widehat{\mathbf{R}}_{e^{*}}^{f}=\frac{\widehat{\mathbf{N}}_{e^{*}}^{H_{f}}-\widehat{\mathbf{N}}_{e^{*}}^{A_{f}}}{\mathbf{B}^{H_{f}}-\mathbf{B}^{A_{f}}}
$$

$$
\widehat{\mathbf{O}}_{e^{*}}^{f}=\frac{\widehat{\mathbf{N}}_{e^{*}}^{A_{f}} * \mathbf{B}^{H_{f}}-\widehat{\mathbf{N}}_{e^{*}}^{H_{f}} * \mathbf{B}^{A_{f}}}{\widehat{\mathbf{N}}_{e^{*}}^{H_{f}}-\widehat{\mathbf{N}}_{e^{*}}^{A_{f}}} .
$$

Apply these coefficients to unfiltered scene data to obtain the forward-scan calibrated radiances

$$
\widehat{\widetilde{\mathbf{L}}}^{C_{f}}=\Re\left\{\frac{\mathbf{N}^{C_{f}}}{\widehat{\mathbf{R}}_{e^{*}}^{f}}-\widehat{\mathbf{O}}_{e^{*}}^{f}\right\} .
$$

The calibrated radiances for reversed scans are computed using the same procedure, and the total combined radiances are written as $\widehat{\widetilde{\mathbf{L}}}^{C}=\left\{\widehat{\widetilde{\mathbf{L}}}^{C_{f}}, \widehat{\widetilde{\mathbf{L}}}^{C_{r}}\right\}$. For the second case, we apply PC filtering to both the calibration and Earth scene data. In this case, the forward-scan radiances can be written as

$$
\widehat{\hat{\mathbf{L}}}_{v}^{C_{f}}=\Re\left\{\frac{\widehat{\mathbf{N}}_{v}^{C_{f}}}{\widehat{\mathbf{R}}_{e^{*}}^{f}}-\widehat{\mathbf{O}}_{e^{*}}^{f}\right\},
$$


where $v$ is the number of eigenvectors used to filter the scene spectra; the optimal value $v^{*}$ is to be determined using an iterative method. The combined radiance matrix for the forward and reverse scans is $\widehat{\widehat{\mathbf{L}}}_{v}^{C}=\left\{\widehat{\hat{\mathbf{L}}}_{v}^{C_{f}}, \widehat{\hat{\mathbf{L}}}_{v}^{C_{r}}\right\}$. Define the difference matrix between these two cases as $\mathbf{D}_{v}=\widehat{\widetilde{\mathbf{L}}}^{C}-\widehat{\hat{\mathbf{L}}}_{v}^{C}$, its RMS value is

$$
d_{v}(k)=\sqrt{\frac{1}{S} \sum_{s=1}^{S}\left[D_{v, s}(k)-\bar{D}_{v}(k)\right]^{2}} .
$$

To derive the optimal number of eigenvectors used by the scene filter, a reference term is generated using the results that were obtained previously. Recall in Section 3.3, the filtered dependent data were applied to filtered independent data in the first case and the filtered dependent data were applied to unfiltered independent data in the third case. Using the results from those two cases, define a new variable to represent the difference between them, for instance,

$\mathbf{D}_{e^{*}}=\widehat{\widetilde{\mathbf{L}}}_{e^{*}}-\widehat{\hat{\mathbf{L}}}_{e^{*}}$. Its RMS value is

$$
d_{r e f}(k)=\sqrt{\frac{1}{T} \sum_{t=1}^{T}\left[D_{e^{*}, t}(k)-\bar{D}_{e^{*}}(k)\right]^{2}},
$$

which represents the minimum noise level experienced by the independent scene data. The optimal number of eigenvectors for the scene filter, $v^{*}$, is the number that leaves a residual slightly smaller than this noise level. It can be determined from

$$
v^{*}=\min _{v}\left|d_{v}(k)-d_{r e f}(k)\right| \forall k .
$$

Figure 5 illustrates the iterative process for finding the optimal number of eigenvectors of the scene filter. Using the reference term $d_{r e f}(k)$ (shown in black), the solution of Eqn. (35) is found so that the residual noise $d_{v}(k)$ (shown in magenta) is similar to the reference noise level. In Figure 6, the absolute summation of $d_{v}(k)-d_{r e f}(k)$ over the entire frequency band, $\sum_{k}\left|d_{v}(k)-d_{r e f}(k)\right|$, is computed at various PC numbers. The optimal PC number is shown as the minimum point on the curve.

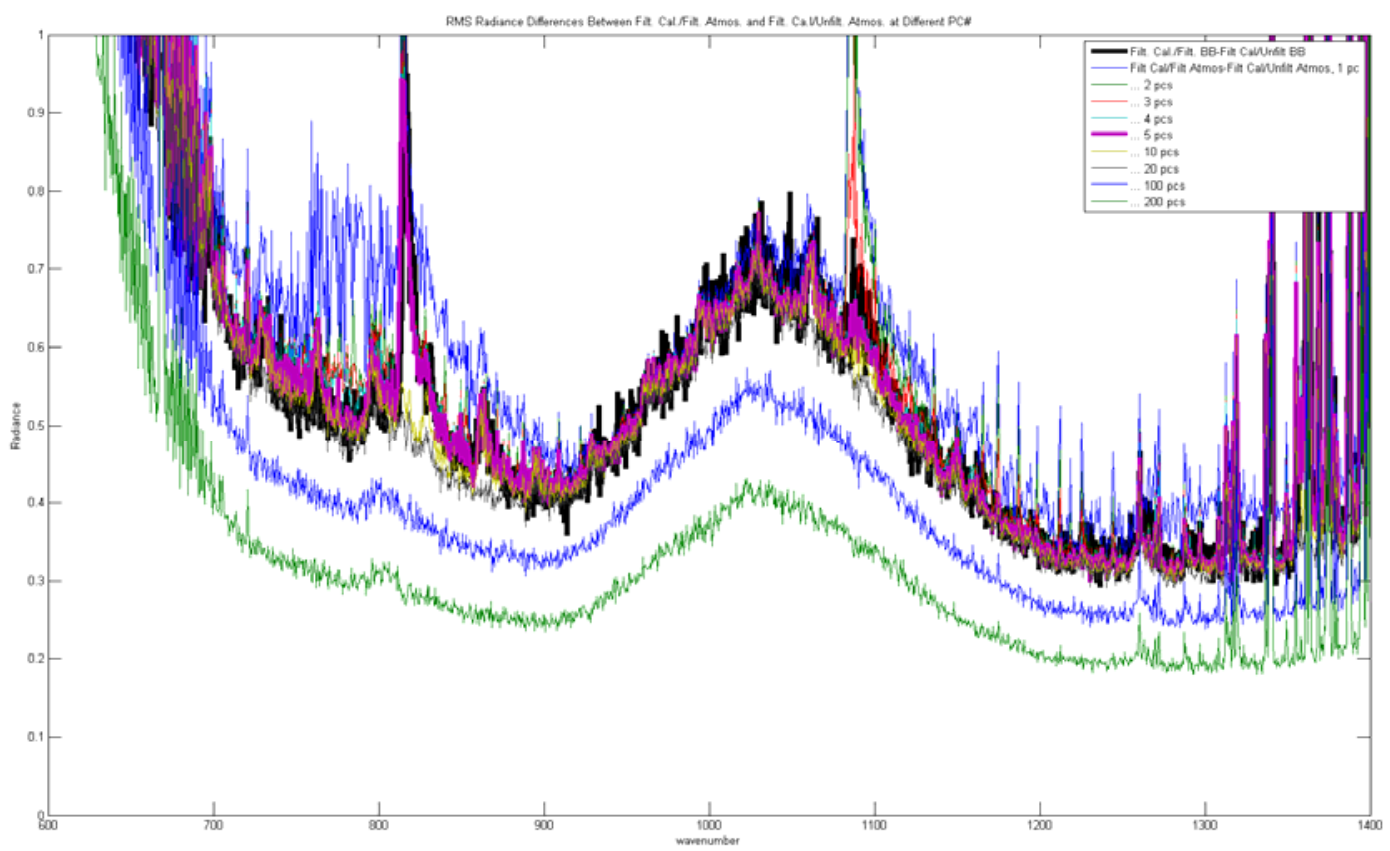

Figure 5. An iterative method for finding the optimal number of eigenvectors used by the scene filter. 


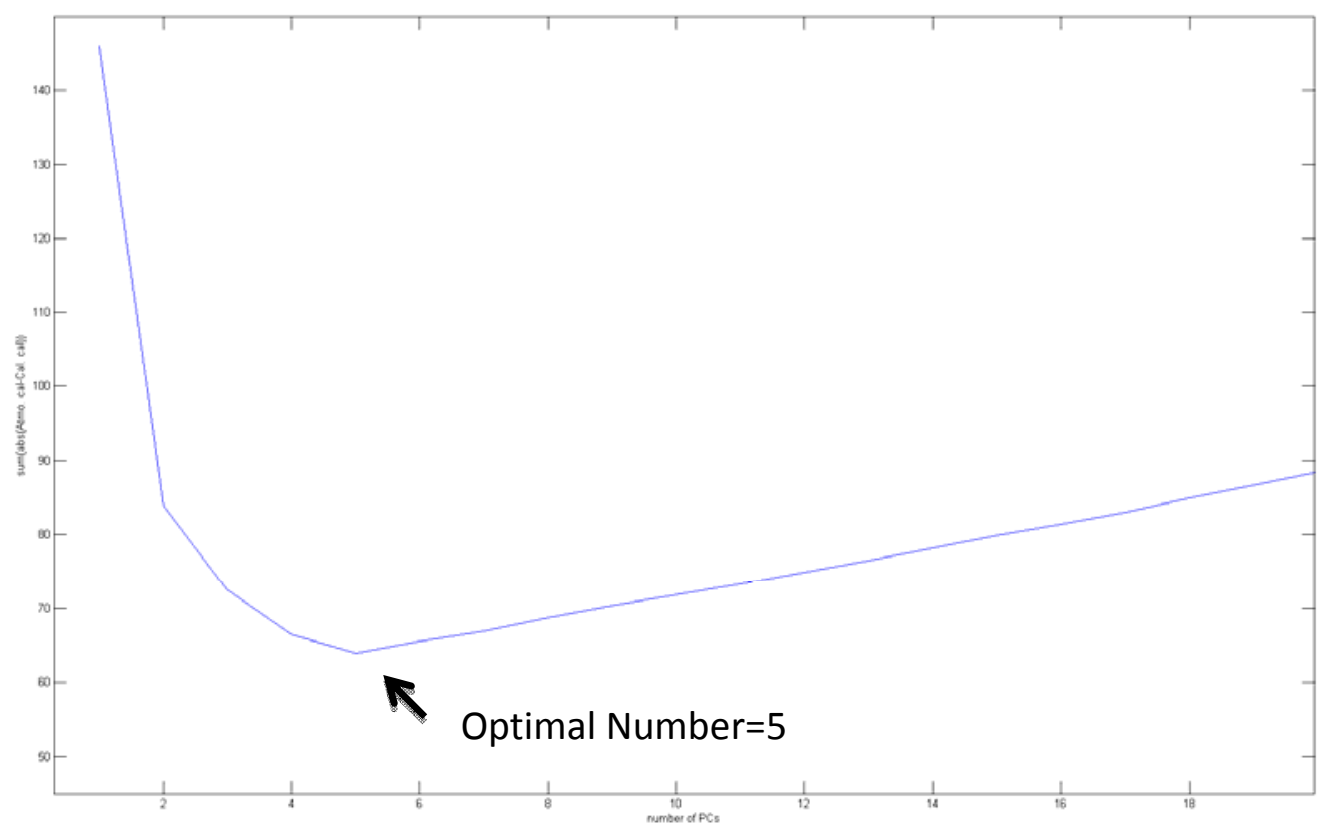

Figure 6. The optimal number of eigenvectors used by the scene filter.

\subsection{Complex Radiometric Calibration Algorithm}

Using the PC number for the calibration filter $e^{*}$ and the PC number for the scene filter $v^{*}$, the PC filtered radiances can be obtained from

$$
\widehat{\hat{\mathbf{L}}}_{v^{*}}^{C_{f}}=\Re\left\{\frac{\widehat{\mathbf{N}}_{v^{*}}^{C_{f}}}{\widehat{\mathbf{R}}_{e^{*}}^{f}}-\widehat{\mathbf{O}}_{e^{*}}^{f}\right\}
$$

and

$$
\widehat{\mathbf{L}}_{v^{*}}^{C_{r}}=\Re\left\{\frac{\widehat{\mathbf{N}}_{v^{*}}^{C_{r}}}{\widehat{\mathbf{R}}_{e^{*}}^{r}}-\widehat{\mathbf{O}}_{e^{*}}^{r}\right\} .
$$

Finally, the combined radiance matrix for the forward and reverse scans is $\widehat{\widehat{\mathbf{L}}}_{v^{*}}^{C}=\left\{\widehat{\widehat{\mathbf{L}}}_{v^{*}}^{C_{f}}, \widehat{\widehat{\mathbf{L}}}_{v^{*}}^{C_{r}}\right\}$.

\section{CONCLUSION}

In this paper, we introduce a refined calibration technique that utilizes a PC noise filter to compensate for instrument distortions and artifacts, therefore, further improve the absolute radiometric calibration accuracy. To test the procedure and estimate the PC filter noise performance, we form dependent and independent test samples by using odd and even sets of blackbody spectra. To see the filtering effect on the calibration noise performance and to find the optimal number of eigenvectors, we examine four different scenarios: apply PC filtering to both dependent and independent datasets, apply PC filtering to dependent calibration data only, apply PC filtering to independent data only, and no PC filters. The independent scene radiances are predicted for each case and comparisons are made. The results show significant reduction in noise in the final calibrated radiances with the implementation of the PC filtering algorithm. 


\section{REFERENCES}

[1] J. Tian, W. L. Smith, and M. J. Gazarik, "Geostationary Imaging Fourier Transform Spectrometer Ground Based Measurement (GIFTS GBM) PCA Calibrations and Lunar Emissivity Estimation,” Proc. SPIE Asia-Pacific Remote Sensing, Nov. 2008.

[2] P. Antonelli, et.al., “A principal component noise filter for high spectral resolution infrared measurements,” Journal of Geophysical Research, vol. 109, D23102, 2004. 